\title{
VECTOR REPRESENTATION OF BINARY IMAGES CONTAINING HALFTONE DOTS
}

\author{
Kei KAWAMURA ${ }^{\ddagger}$, Hiroshi WATANABE ${ }^{\ddagger}$, Hideyoshi TOMINAGA ${ }^{\dagger, \ddagger}$
}

\author{
†Waseda University \\ Dept. of Elec., Info. and Comm. Eng. \\ 3-4-1, Okubo, Shinjuku-ku, Tokyo, Japan
}

\section{ABSTRACT}

Vector representation of graphics has an advantage of being displayed at any size. When resolution of a bitmap image is changed, lack of a line segment arises. In addition, moire occurs when resolution of an image with halftone dots is resized. In this paper, we propose a new technique to convert the binary image with halftone dots into the vector representation. Resolution conversion of binary image can easily be performed without moire by continuous tone approximation of halftone dots. First, we separate the area of halftone dots and line drawings in the image. Next, a continuous tone approximation is applied to the area of halftone dots. Then, the conventional vectorization is applied to both continuous tone areas and line drawings. Finally these components are mixed and reconstructed. Our approach provides an efficient way of displaying cartoon like images at any size with limited amount of data.

\section{INTRODUCTION}

A facsimile document or a newspaper has been printed on a paper as a hard copy. On the other hand, demands of the soft copy which can be displayed on a monitor at any size have been increasing. This trend has been accelerated by the dissemination of personal computers and portable devices. Moreover, pervasion of low cost scanners has provided the environment where users can scan images easily.

In most cases, facsimile documents and newspapers are binary images. Offset printing also produces a manuscript with binary representation by ink dots on a paper. Although binary images cannot represent gray scale, it has an advantage of being compressed with smaller data size.

The resolution of a monitor is normally low compared to the one of a scanner. For this reason, in order to display the image obtained from a scanner, resolution conversion becomes indispensable. However, jaggy is often observed when a curve or a slash line is expanded. Considering the above problem, it seems reasonable to take an approach that changes the binary image into a vector representation before resizing.

\author{
$\ddagger$ Waseda University \\ Graduate School of GITS \\ 1-3-10, Nishiwaseda, Shinjuku-ku, Tokyo, Japan
}

The purpose of this paper is to carry out resolution conversion of binary image easily. We propose a new technique to describe a binary image containing halftone dots by a vector representation. For this description, halftone dots are approximated by a continuous tone, which corresponds to the dot density. In Section 2, we show results of a preliminary experiment. In Section 3, the proposed approach is described. Finally simulation results are shown in Section 4 , and the conclusion is given in Section 5 .

\section{PRELIMINARY EXPERIMENT}

\subsection{Vector Conversion Tools}

Bezier and B-spline curves are typical parametric curves that can describe shapes. There are many softwares to convert raster images to vector graphics. AutoTrace[1] is one of such softwares using Bezier curves representation. We use this software for vector conversion since it provides quite precise approximation. An error threshold $e$ is prepared in AutoTrace as one of input parameters, and its default value of $e$ is 2.0 .

We investigated the relation of this error threshold and EPS file size obtained by AutoTrace. The results are shown in Fig.1. It is turned out that file size is in inverse proportion to the value of $e$.

\subsection{Simple Vector Conversion}

We convert a printed paper image of a $12.8 \mathrm{~cm} \times 18.2 \mathrm{~cm}$ (5inch $\times 7$ inch) size, which is scanned as a binary image, using AutoTrace by changing of scan resolution and the error threshold. AutoTrace outputs an EPS file. However, the EPS file format is too redundant in terms of data compression. Thus, this is converted to a Flash file format[2]. The relation of scan resolution, the Flash file size, and the error threshold are shown in top three series of Fig.2.

This results show that the file size is proportional to scan resolution over 300dpi. However, when scan resolution is $200 \mathrm{dpi}$ or less, the file size becomes small rapidly. Thus, we observed the image at $150 \mathrm{dpi}$, and found that most halftone 


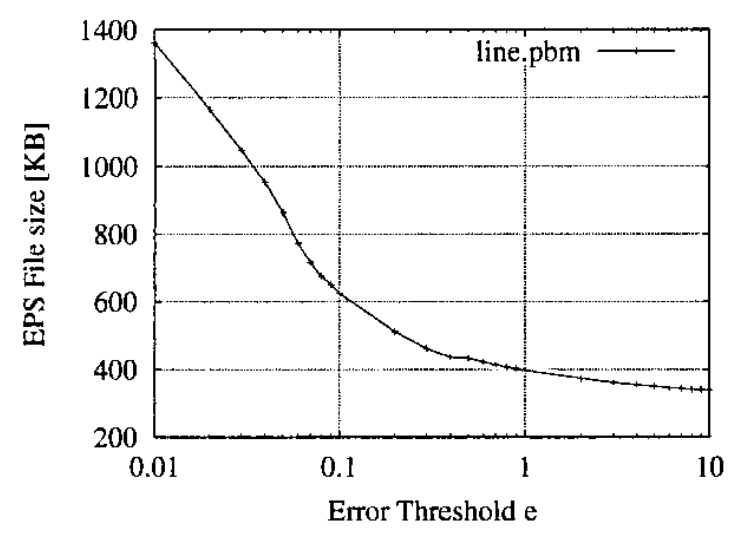

Fig. 1. The relation of the error threshold $e$ and the file size

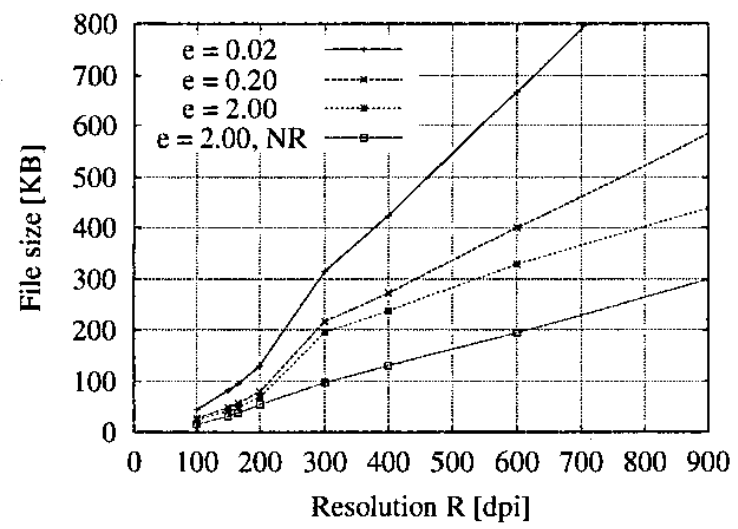

Fig. 2. The relations of scan resolution, the file size, the error threshold, and a noise reducer (NR)

dots are turned to a white background and only line drawings can be seen. This means that most halftone dots are lost at low resolution.

Jaggy is reduced when the image is zoomed in because of vector representation. However, moire still occurs, so that the simple vector conversion does not provide a solution to this problem.

\subsection{Vector Conversion with Noise Reducer}

Next, we consider halftone dots removal positively. For this purpose, we use a noise reducer since they can be regarded as a set of isolated small areas. The relation of scan resolution, and the Flash file size using noise reducer is shown in the bottom series of Fig.2.

This result shows the following two consequences. First, removing halftone dots is effective for archiving binary im-

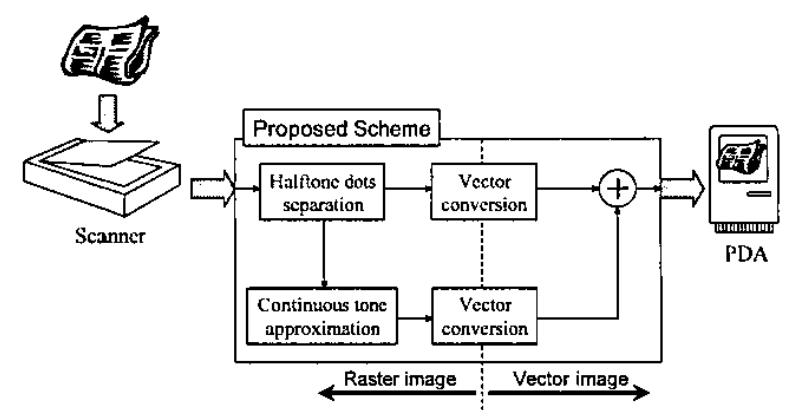

Fig. 3. Conversion system with proposed scheme

ages. Second, vector representation is suitable for line drawings [3], but not for halftone dots.

Thus, removal or separation of halftone dots is an effective approach to convert binary bitmap image to vector representation. However, an image only with line drawings lacks the reproducibility. Therefore, halftone dots representation should be changed to others such as continuous tone which is suitable for vector representation[4].

\section{PROPOSED VECTOR CONVERSION SCHEME}

\subsection{Conversion System}

We propose a new technique to convert binary images containing halftone dots to the one suitable for vector representation. A conversion system with a proposed scheme is illustrated by a diagram in Fig. 3 .

We assume that a printed material is input to a scanner as binary image, and converted to vector representation by the proposed scheme. Then, the file is viewed on a monitor of PC or on a panel of PDA.

First, we separate an area of halftone dots and line drawings in the image. Next, a continuous tone approximation is applied to the area of halftone dots. Then, the conventional vectorization is applied to both a continuous tone image and line drawings. Finally, these two components are mixed together.

\subsection{Halftone Dots Separation}

We use two characteristics of halftone dots for their separation. It is an isolated small area where tone value is low or high. Where the tone value of halftone dots area is medium, it would be changed by a closing operation, whereas it remains the same if the same area is represented in line drawings. A halftone dots area is detected by a dilation operation to the halftone dots.

First, we search connected pixels which are isolated small area. Then we remove them as halftone dots if small area 
is smaller than a threshold. Next, the closing operation is performed to the residual image. If a ratio of changed pixels in the surroundings of the connected pixel is over than a threshold, the connected pixel is regarded as halftone dots. Then we can detect halftone dots where tone value is low or medium.

The process described above is also applied to an inverse input image, in which black and white pixels are inverted. By this operation, inverse halftone dots are detected where tone value is originally high. Finally, we get line drawings, halftone dots, inverse halftone dots, and halftone dots areas.

\subsection{Continuous Tone Approximation}

We calculate tone value for each halftone dots area. Now we process on an assumption that halftone dots represent a homogeneous or a linear gradation tone. For example, comic images using screen tones or office documents containing simple shapes fulfill the assumption. If a bitmap image is three-dimensional data, then homogeneous and linear gradation tones would be represented by plane equation.

Thus, we divide halftone dots area into some small areas. Next, we calculate tone values as constant in each small area. The tone value is defined by a ratio of black pixels to the small area. Then, three parameters of plane equation are detected using tone values and positions of small areas by least-square method.

\subsection{Vector Conversion}

Line drawings and continuous tone approximated images are converted to vector representation. For the vector conversion, AutoTrace is used since it provides quite good approximation. An output file format of AutoTrace is EPS. On the other hand, Flash can provide quite handy representation for PDA. Thus, we convert the EPS to the Flash file format by Illustrator[5]. A mixing process of them can also be done by Illustrator.

\section{EXPERIMENT AND EVALUATION}

\subsection{Implementation}

Simulation experiment is carried out to confirm the proposed method. An image using in this experiment is a one page of comics. An overall view of the input image is shown in Fig. 4 and a small part of this is shown in Fig.5. The image is scanned at $300 \mathrm{dpi}$ as a binary image.

For halftone dots separation, an isolated small area is removed if its size is less than 7 pixels. In addition, if a ratio of changed pixels in the surroundings is $80 \%$ or over, such area is regarded as halftone dots. These thresholds are detected through some experiments.

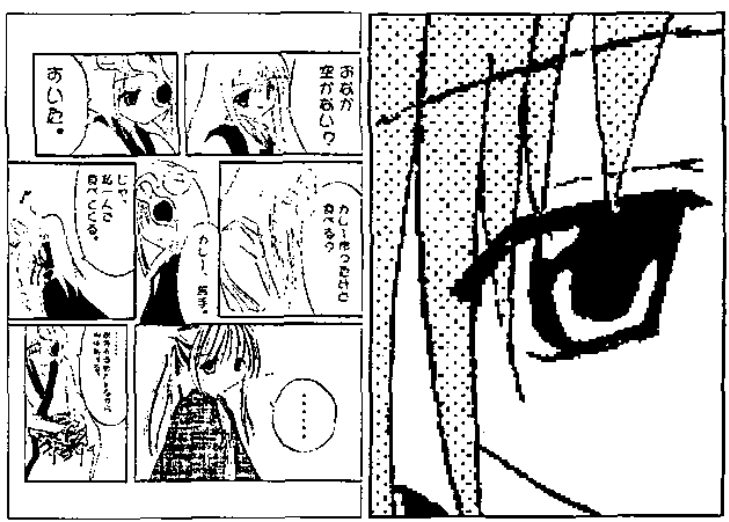

Fig. 4. Original Image

Fig. 5. Input

\subsection{Conversion Result Images}

The result images of halftone dots separation are presented in Fig.6 and Fig.7. The continuous tone approximation image is presented in Fig.8. The mixed image of line drawings and the continuous tone approximation in raster representation is presented in Fig.9.

A vector representation can be obtained by converting above images using AutoTrace. The error threshold $e$ is set to the default value. Viewing that by Flash player, it is confirmed that jaggy and moire are not occurred.

Through this experiment, it is confirmed that our proposed method basically work out. On the other hand, it becomes obvious that very small lines are separated to half tone dots. Moreover, thresholds are not robust for scan resolution and screen ruling ${ }^{1}$.

\subsection{Evaluation}

Our approach could be evaluated from two perspective, halftone dots separation and continuous tone approximation. Here, we focus on the latter.

The evaluation method of an approximation is the following. First, we generate continuous tone images by mathematical programming. Next, these images are converted to binary images by a halftone dots method. It must be noted that image size, image resolution, and screen ruling are related. Then, our proposed method is applied for them and tone parameters are detected. Finally, we checked the difference between original and estimated tone parameters.

The estimation result on homogeneous halftone dots is shown in Fig.10. In the figure, experimentations are carried out for $60 \mathrm{lpi}$ and $85 \mathrm{lpi}$, and for 45 degree or 90 degree of screen angles. It is confirmed that detected tone values lie

\footnotetext{
${ }^{1}$ The frequency of a row of dots in a halftone image, usually expressed as lines per inch (lpi).
} 


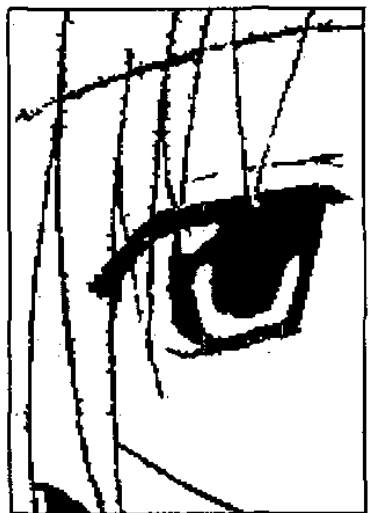

Fig. 6. Line drawings

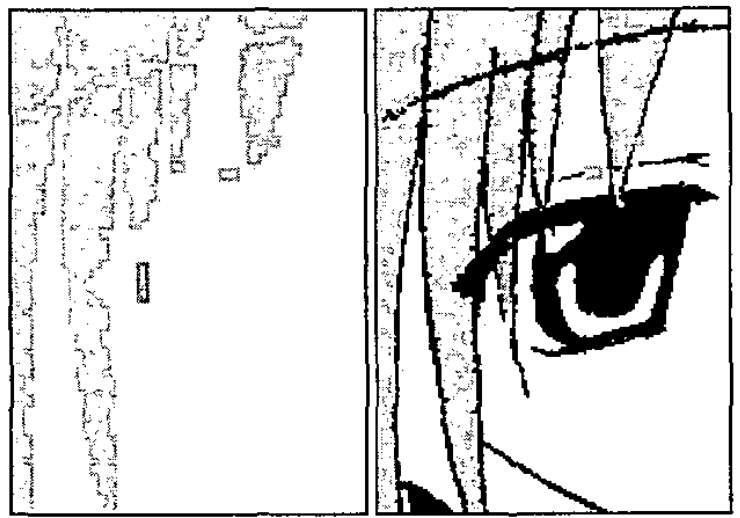

Fig. 8. Continuous tone approximation

on theoretical values mostly independently screen ruling or screen angle. However, in some cases, tone values are detected $10 \%$ lower than theoretical one. It is considered to be related that a shape of cluster of dots changes when the density is from $40 \%$ to $50 \%$.

\section{CONCLUSION}

In this paper, we propose the new technique to convert the binary image with halftone dots into the vector representation. First, we separate the area of halftone dots and line drawings in the image. Next, the continuous tone approximation is applied to the area of halftone dots. Then, the conventional vectorization is applied to both continuous tone areas and line drawings. Finally these components are mixed and reconstructed. Through the experiment, we could confirm the validity of the proposed method.

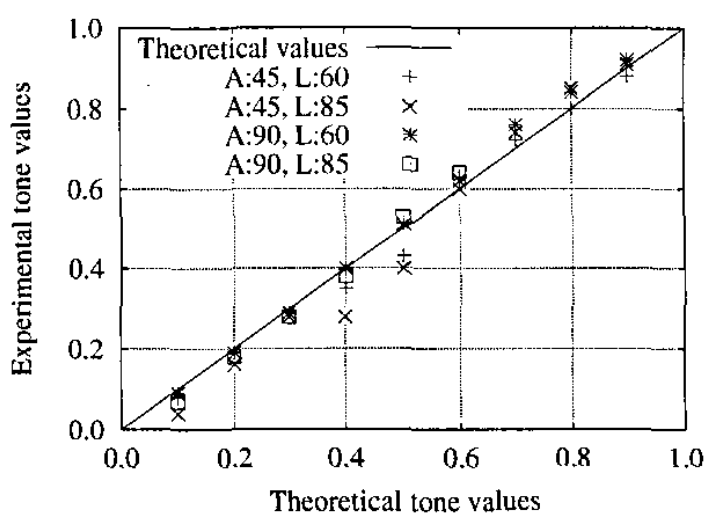

Fig. 10. The relations of theoretical and experimental tone values on a homogeneous halftone dots

\section{REFERENCES}

[1] "AutoTrace,"

http: //autotrace. sourceforge.net/

[2] "Macromedia Flash File Format (SWF) Specification," http: //www. macromedia.com/software /flash/open/Iicensing/fileformat/

[3] O. Nakagami, T. Miyazawa, H. Watanabe, H. Tominaga, "A Study on Two-layer Coding for Animation Images," IEEE Int. Conf. on Multimedia Expo (ICME) 2002, WedAmPO3: Compression II, Aug. 2002.

[4] K. Kawamura, H. Watanabe, H. Tominaga, "A study on Vector Representation of Binary Images Containing Halftone Dots," Picture Coding Symposium of Japan (PCSJ) 2003, P-2.13, Nov. 2003.

[5] "Illustrator," http: / /www. adobe.co.jp/ 